International Journal of Pure and Applied Mathematics

Volume 107 No. 1 2016, 161-177

ISSN: 1311-8080 (printed version); ISSN: 1314-3395 (on-line version)

url: http://www.ijpam.eu

doi: 10.12732/ijpam.v107i1.13

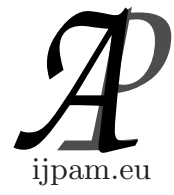

\title{
NEW JULIA AND MANDELBROT SETS FOR \\ A NEW FASTER ITERATIVE PROCESS
}

\author{
Mandeep Kumari ${ }^{1}$, Ashish $^{2}$, Renu Chugh ${ }^{3}$ \\ ${ }^{1,3}$ Department of Mathematics \\ Maharshi Dayanand University \\ Rohtak, 124001, INDIA \\ ${ }^{2}$ Department of Mathematics \\ RPS Degree College, Balana \\ Mahendergarh, 123029, INDIA
}

\begin{abstract}
Fixed point iterative procedures are the backbones of fractal geometry. In existing literature Julia sets, Mandelbrot sets and their variants have been studied using one - step, two - step, three - step and four - step iterative process. Recently, M. Abbas and T. Nazir [12] introduced a new iterative process (a four-step iterative process) which is faster than all of Picard, Mann and Agarwal processes. In this paper, we obtain further generalizations of Julia and Mandelbrot sets using this faster iterative process for quadratic, cubic and higher degree polynomials. Further, we analyze that few Julia and Mandelbrot sets took the shape of Lord Ganesha (name of Hindu God), Dragon and Urn.
\end{abstract}

AMS Subject Classification: 37F45, 37F50

Key Words: Julia set, Mandelbrot set, four-step feedback process, escape criterion, complex polynomials.

\section{Introduction}

Complex analytic dynamics attains a landmark in the field of fractal theory during the time of great French mathematician Gaston Julia. Julia set is of vital importance in the study of the complex dynamics of functions because it is a place where the chaotic behavior of complex functions occur [10]. In 1975,

\footnotetext{
Received: December 29, 2015

(c) 2016 Academic Publications, Ltd.

Published: March 26, 2016

url: www.acadpubl.eu
}

$\S$ Correspondence author 
Mandelbrot extended the work of Gaston Julia and introduced Mandelbrot set. During that time, various Julia and Mandelbrot sets have been introduced for quadratic $[3,21,24,10]$, cubic $[21,1,2,11,22]$ and higher degree polynomials [26] using Picard iterative process (a one step feedback process) [21].

Many researchers have studied Julia and Mandelbrot sets from different aspects. In 2009, D. Rochon [9] studied generalized Mandelbrot sets in bicomplex plane. Later on the work of Rochon was extended by Wang [25] jointly with others and carried further analysis of generalized Julia and Mandelbrot sets. Also, they have studied the fractal structure and discontinuity law of the generalized Julia sets generated from the extended complex mapping $z^{n}+c$, where $n \in R$ [25]. Further, Julia and Mandelbrot sets have been studied under the effect of noises $[19,4,14,15,27]$. In 2004, Rani and Kumar [17, 18] introduced the superior iterate and created superior Julia and Mandelbrot sets for quadratic $[17,18]$ and cubic $[17,18]$ polynomials. Later on, they also presented Julia and Mandelbrot sets for higher $[13,16]$ degree complex polynomials. Further, in 2008, Rani and Negi [5] studied the midgets of Mandelbrot sets. In 2011, Singh, Mishra and Sinkala [23] presented a complete literature review of superior fractals existing in the literature, see also[20].

Recently, Ashish et al. [8] used Noor iterative process (an example of fourstep feedback process) for creating new Julia and Mandelbrot sets for quadratic, cubic and higher degree polynomials. Further they have shown anti Julia, Multicorns [6] and cubic Julia sets [7] using Noor iterative process.

In this paper, we create Julia and Mandelbrot sets using a new faster iterative process [12], which is an example of a four-step feedback process. In Section 2, we give some definitions, which are the basis of our work. In Section 3 , we discuss escape criterions for quadratic, cubic and nth degree polynomials under the new iteration process. Several Julia and Mandelbrot sets have been presented in Sections 4 and 5 respectively. Finally, the paper has been concluded in Section 6.

\section{Preliminaries}

In 2014, M. Abbas and T. Nazir [12] introduced the following new iterative process which is faster than all of Picard, Mann and Agarwal et al. processes and is independent of these iterative processes.

Definition 2.1. Let us consider a sequence $\left\{x_{n}\right\}$ of iterates for initial point $x_{0} \in X$ such that

$$
\left\{x_{n+1}: x_{n+1}=\left(1-\alpha_{n}\right) T y_{n}+\alpha_{n} T z_{n} ;\right.
$$




$$
\begin{array}{r}
y_{n}=\left(1-\beta_{n}\right) T x_{n}+\beta_{n} T z_{n} \\
z_{n}=\left(1-\gamma_{n}\right) x_{n}+\gamma_{n} T x_{n} \\
n=0,1,2, \ldots,\}
\end{array}
$$

where $\alpha_{n}, \beta_{n}, \gamma_{n} \in[0,1]$ and $\left\{\alpha_{n}\right\},\left\{\beta_{n}\right\},\left\{\gamma_{n}\right\}$ are sequences of positive numbers. For the sake of simplicity, we take $\alpha_{n}=\alpha, \beta_{n}=\beta$ and $\gamma_{n}=\gamma$.

Definition 2.2. (Julia Set). The filled in Julia set of the function $Q$ is defined as

$$
K(Q)=\left\{z \in C: Q^{k}(z) \text { does not tend to infinity }\right\}
$$

where $C$ is the complex space, $Q^{k}(z)$ is $k^{\text {th }}$ iterate of function $Q$ and $K(Q)$ denotes the filled Julia set. The Julia set of the function $Q$ is defined to be the boundary of $K(Q)$, i.e.,

$$
J(Q)=\alpha K(Q)
$$

where $J(Q)$ denotes the Julia set. The set of points whose orbits are bounded under the Picard orbit $Q_{c}(z)=z^{2}+c$ is called the Julia set. We choose the initial point 0 , as 0 is the only critical point of $Q_{c}[21]$.

Definition 2.3. (Mandelbrot Set). The Mandelbrot set $M$ consists of all parameters $c$ for which the filled Julia set of $Q_{c}$ is connected, that is

$$
M=\left\{c \in C: K\left(Q_{c}\right) \text { is connected }\right\}
$$

In fact, $M$ contains an enormous amount of information about the structure of Julia sets. The Mandelbrot set $M$ for the Quadratic $Q_{c}(z)=z^{2}+c$ is defined as the collection of all $c \in C$ for which the orbit of the point 0 is bounded, that is

$$
M=\{c \in C ; n=0,1,2, \ldots\} \text { is bounded. }
$$

We choose the initial point 0 as 0 is the only critical point of $Q_{c}$ [21].

\section{Escape Criterions for Complex Polynomials using Faster Iterative Process}

The escape criterion plays a vital role in the generation and analysis of Julia sets, Mandelbrot sets and their variants. We need the following escape criterions for the quadratic, cubic and higher degree polynomials. 


\subsection{Escape Criterions for Quadratic Polynomials}

Throughout this section, we assume that $T\left(x_{n}\right)=Q_{c}^{\prime \prime}(z), T\left(z_{n}\right)=Q_{c}^{\prime}(z)$ and $T\left(y_{n}\right)=Q_{c}(z)$.

Theorem 3.1. Suppose $|z| \geq|c|>2 / \alpha,|z| \geq|c|>2 / \beta$ and $|z| \geq|c|>$ $2 / \gamma$, where $0<\alpha<1,0<\beta<1$ and $0<\gamma<1$ and $c$ is a complex number. Define

$$
\begin{aligned}
& z_{1}=\alpha Q_{c}(z)+(1-\alpha) z, \\
& z_{2}=\alpha Q_{c}\left(z_{1}\right)+(1-\alpha) z_{1}, \\
& \ldots, \\
& \ldots, \\
& \ldots, \\
& z_{n}=\alpha Q_{c}\left(z_{n-1}\right)+(1-\alpha) z_{n-1},
\end{aligned}
$$

where $Q_{c}(z)$ can be a quadratic, cubic or biquadratic polynomial in terms of $\gamma$ and $n=2,3, \ldots$ then $\left|z_{n}\right| \rightarrow \infty$ as $n \rightarrow \infty$.

Proof. For $Q_{c}^{\prime \prime}(z)=z^{2}+c$, we consider

$$
\begin{aligned}
\left|Q_{c}^{\prime}(z)\right| & =\left|(1-\gamma) z+\gamma Q_{c}^{\prime \prime}(z)\right| \\
& =\left|\gamma z^{2}+(1-\gamma) z+\gamma c\right| \\
& \geq\left|\gamma z^{2}+(1-\gamma) z\right|-|\gamma c| \\
& \geq|z|(|\gamma z+(1-\gamma)|)-\gamma|z| \quad(\text { since }|z| \geq|c|) \\
\left|Q_{c}^{\prime}(z)\right| & \geq|z|(|\gamma z|-1) .
\end{aligned}
$$

Also

$$
\begin{aligned}
\left|Q_{c}(z)\right| & =\left|(1-\beta) Q_{c}^{\prime \prime}(z)+\beta Q_{c}^{\prime}(z)\right| \\
& =\left|(1-\beta)\left(z^{2}+c\right)+\beta\right| z|(|\gamma z|-1)| \\
& =\left.|\beta \gamma| z\right|^{2}+(1-\beta) z^{2}+(1-\beta) c-\beta|z| \mid \\
& \geq \beta \gamma|z|^{2}+\left|(1-\beta) z^{2}\right|-(1-\beta)|z|-\beta|z| \\
& \geq \beta \gamma|z|^{2}+(1-\beta)\left|z^{2}\right|-|z|+\beta|z|-\beta|z| \\
& \geq|z|[(\beta \gamma-\beta+1)|z|-1] \\
\text { i.e. }\left|Q_{c}(z)\right| & \geq|z|[(\beta \gamma-\beta+1)|z|-1] .
\end{aligned}
$$

Now, we have

$$
z_{n}=(1-\alpha) T y_{n}+\alpha T z_{n}
$$




$$
\begin{aligned}
& \left|z_{n}\right|=\left|(1-\alpha) Q_{c}(z)+\alpha Q_{c}^{\prime}(z)\right| \\
& \left|z_{1}\right|=\left|(1-\alpha) Q_{c}(z)+\alpha Q_{c}^{\prime}(z)\right|
\end{aligned}
$$

On substituting (3.1) and (3.2) in (3.3), we get

$$
\begin{aligned}
&\left|z_{1}\right|=|(1-\alpha)[|z|[(\beta \gamma-\beta+1)|z|-1]]+\alpha| z|(\gamma|z|-1)| \\
&\left.=\left.|(|z|-\alpha|z|)[(\beta \gamma-\beta+1)|z|-1]+\alpha \gamma| z\right|^{2}-\alpha|z|\right) \mid \\
& \geq\left.|\beta \gamma| z\right|^{2}-\beta|z|^{2}+|z|^{2}-\alpha \beta \gamma|z|^{2}+\alpha \beta|z|^{2}-\alpha|z|^{2} \\
&-|z|+\alpha|z|+\alpha \gamma|z|^{2}-\alpha|z| \mid \\
& \geq|z|[\beta \gamma|z|-\beta|z|+|z|-\alpha \beta \gamma|z| \\
&+\alpha \beta|z|-\alpha|z|-1+\alpha \gamma|z|] \\
& \geq|z|[(\alpha \beta+\beta \gamma+\alpha \gamma-\alpha \beta \gamma-\alpha-\beta+1)|z|-1] \\
& \text { i.e. } \quad\left|z_{1}\right| \geq|z|[(\alpha \beta+\beta \gamma+\alpha \gamma-\alpha \beta \gamma-\alpha-\beta+1)|z|-1]
\end{aligned}
$$

Since $|z| \geq|c|>2 / \alpha,|z| \geq|c|>2 / \beta$ and $|z| \geq|c|>2 / \gamma$, so that $|z|>2 / \alpha \beta \gamma>2 /(\alpha \beta+\beta \gamma+\alpha \gamma-\alpha \beta \gamma-\alpha-\beta+1)$. Therefore there exists $\lambda>0$, such that $(\alpha \beta+\beta \gamma+\alpha \gamma-\alpha \beta \gamma-\alpha-\beta+1)|z|-1>1+\lambda$, consequently

$$
\left|z_{1}\right|>(1+\lambda)|z|
$$

In particular, $\left|z_{n}\right|>|z|$. So we may apply the same argument repeatedly to find

$$
\left|z_{n}\right|>(1+\lambda)^{n}|z|
$$

Thus, the orbit of $z$ tends to infinity. This completes the proof.

Corollary 3.2. Suppose that $|c|>2 / \alpha,|c|>2 / \beta$ and $|c|>2 / \gamma$, then the orbit $O\left(Q_{c}, 0, \alpha, \beta, \gamma\right)$ escapes to infinity.

The proof of the escape criterion actually gives us a little more information. In the proof, we used the only fact that $|z|>|c|$ and $|c|>2 / \alpha,|c|>2 / \beta$ and also $|c|>2 / \gamma$. Hence we have the following refinement of the escape criterion:

Corollary 3.3. (Escape Criterion). Let $|z|>\max \{|c|, 2 / \alpha, 2 / \beta, 2 / \gamma\}$, then $\left|z_{n}\right|>(1+\lambda)^{n}|z|$ as $\left|z_{n}\right| \rightarrow \infty$ as $n \rightarrow \infty$.

We observe that if $\left|z_{k}\right|>\max \{|c|, 2 / \alpha, 2 / \beta, 2 / \gamma\}$ for some $k \geq 0$, then we may apply Corollary 3.2 to $\left|z_{k}\right|$ to have the following result. 
Corollary 3.4. Let for some $k \geq 0$, we have $\left|z_{k}\right|>\max \{|c|, 2 / \alpha, 2 / \beta, 2 / \gamma\}$. Then $\left|z_{k+1}\right|>(1+\lambda)^{n}\left|z_{k}\right|$ so that $\left|z_{n}\right| \rightarrow \infty$ as $n \rightarrow \infty$.

Note that this corollary gives us an algorithm for computing the filled Julia set of quadratics for any complex constant $c$. For any point $z$ which satisfies $|z| \leq|c|$, we find out the orbit of $z$. If, for any $n,\left|z_{n}\right|$ lies outside the circle of radius $\max \{|c|, 2 / \alpha, 2 / \beta, 2 / \gamma\}$, then we say that the orbit escapes to infinity that means $z$ is not in the filled Julia set. On the other hand, if $\left|z_{n}\right|$ never exceeds this bound, then $z$ is by definition in Julia set.

\subsection{Escape Criterion for Cubic Polynomials}

We consider the following form of a cubic polynomial as it is conjugate to all the cubic polynomials:

$$
C_{c}(z)=z^{3}+c, \text { where } c \text { are complex numbers. }
$$

Also, throughout this section, we assume that $T\left(x_{n}\right)=C_{c}^{\prime \prime}(z), T\left(z_{n}\right)=$ $C_{c}^{\prime}(z)$ and $T\left(y_{n}\right)=C_{c}(z)$.

Theorem 3.5. Suppose $|z| \geq|c|>(2 / \alpha)^{1 / 2},|z| \geq|c|>(2 / \beta)^{1 / 2}$ and $|z| \geq|c|>(2 / \gamma)^{1 / 2}$, where $0<\alpha<1,0<\beta<1$ and $0<\gamma<1$ and $c$ is a complex number. Define

$$
\begin{aligned}
& z_{1}=\alpha C_{c}(z)+(1-\alpha) z, \\
& z_{2}=\alpha C_{c}\left(z_{1}\right)+(1-\alpha) z_{1}, \\
& \ldots, \\
& \ldots, \\
& \ldots, \\
& z_{n}=\alpha C_{c}\left(z_{n-1}\right)+(1-\alpha) z_{n-1},
\end{aligned}
$$

where $C_{c}(z)$ is a polynomial in terms of $\gamma$ and $n=2,3, \ldots$ then $\left|z_{n}\right| \rightarrow \infty$ as $n \rightarrow \infty$.

Proof. For $C_{c}^{\prime \prime}(z)=z^{3}+c$, we have considered

$$
\begin{aligned}
\left|C_{c}^{\prime}(z)\right| & =\left|(1-\gamma) z+\gamma C_{c}^{\prime \prime}(z)\right| \\
& =\left|\gamma z^{3}+(1-\gamma) z+\gamma c\right| \\
& \geq \gamma|z|^{3}-(1-\gamma)|z|-\gamma|c| \\
\text { i.e. }\left|C_{c}^{\prime}(z)\right| & \geq|z|\left(\gamma|z|^{2}-1\right) .
\end{aligned}
$$


Also

$$
\begin{aligned}
\left|C_{c}(z)\right| & =\left|(1-\beta) C_{c}^{\prime \prime}(z)+\beta C_{c}^{\prime}(z)\right| \\
& \geq\left|(1-\beta)\left(z^{3}+c\right)+\beta\right| z\left|\left(\gamma|z|^{2}-1\right)\right| \\
& \geq(1-\beta)|z|^{3}-(1-\beta)|c|+\beta \gamma|z|^{2}-\beta|z| \\
& \geq|z|\left[(\beta \gamma-\beta+1)|z|^{2}-1\right] \\
\text { i.e. } \quad\left|C_{c}(z)\right| & \geq|z|\left[(\beta \gamma-\beta+1)|z|^{2}-1\right] .
\end{aligned}
$$

Now, we have

$$
\begin{aligned}
& \left|z_{n}\right|=\left|(1-\alpha) C_{c}(z)+\alpha C_{c}^{\prime}(z)\right| \\
& \left|z_{1}\right|=\left|(1-\alpha) C_{c}(z)+\alpha C_{c}^{\prime}(z)\right|
\end{aligned}
$$

Using (3.5) and (3.6), we get

$$
\begin{aligned}
\left|z_{1}\right| & \geq \mid\left(1-\alpha\left[|z|\left((\beta \gamma-\beta+1)|z|^{2}-1\right)\right]+\alpha\left[|z|\left(\gamma|z|^{2}-1\right)\right] \mid\right. \\
& \geq|z|\left[(\alpha \beta+\beta \gamma+\alpha \gamma-\alpha \beta \gamma-\alpha-\beta+1)|z|^{2}-1\right] \\
\text { i.e. }\left|z_{1}\right| & \geq|z|\left[(\alpha \beta+\beta \gamma+\alpha \gamma-\alpha \beta \gamma-\alpha-\beta+1)|z|^{2}-1\right]
\end{aligned}
$$

Since $|z| \geq|c|>(2 / \alpha)^{1 / 2},|z| \geq|c|>(2 / \beta)^{1 / 2}$ and $|z| \geq|c|>(2 / \gamma)^{1 / 2}$, so that $|z|>(2 / \alpha \beta \gamma)^{1 / 2}>(2 /(\alpha \beta+\beta \gamma+\alpha \gamma-\alpha \beta \gamma-\alpha-\beta+1))^{1 / 2}$. Therefore there exists $\lambda>0$, such that $(\alpha \beta+\beta \gamma+\alpha \gamma-\alpha \beta \gamma-\alpha-\beta+1)|z|^{2}-1>1+\lambda$, consequently

$$
\left|z_{1}\right|>(1+\lambda)|z|
$$

In particular, $\left|z_{n}\right|>|z|$. So we may apply the same argument repeatedly to find

$$
\left|z_{n}\right|>(1+\lambda)^{n}|z|
$$

Thus, the orbit of $z$ tends to infinity. This completes the proof.

Corollary 3.6. (Escape Criterion). Let

$$
|z|>\max \left\{|c|,(2 / \alpha)^{1 / 2},(2 / \beta)^{1 / 2},(2 / \gamma)^{1 / 2}\right\},
$$

then $\left|z_{n}\right|>(1+\lambda)^{n}|z|$ as $\left|z_{n}\right| \rightarrow \infty$ as $n \rightarrow \infty$. 


\subsection{Escape Criterion for $n^{\text {th }}$ Degree Polynomials}

Now, we discuss the general escape criterion for polynomials of the form

$$
G_{c}(z)=z^{n}+c, \text { where } c \text { is a complex numbers. }
$$

Also taking $T\left(x_{n}\right)=G_{c}^{\prime \prime}(z), T\left(z_{n}\right)=G_{c}^{\prime}(z)$ and $T\left(y_{n}\right)=G_{c}(z)$.

Theorem 3.7. Let $G_{c}(z)=z^{n}+c, n=1,2,3 \ldots$ be a general polynomial, where $0<\alpha<1,0<\beta<1$ and $0<\gamma<1$ and $c$ is a complex number. Define

$$
\begin{aligned}
& z_{1}=\alpha G_{c}(z)+(1-\alpha) z, \\
& z_{2}=\alpha G_{c}\left(z_{1}\right)+(1-\alpha) z_{1}, \\
& \ldots, \\
& \ldots, \\
& \ldots, \\
& z_{n}=\alpha G_{c}\left(z_{n-1}\right)+(1-\alpha) z_{n-1},
\end{aligned}
$$

where $n=2,3, \ldots$ then $\max \left(|c|,(2 / \alpha)^{1 / n-1},(2 / \beta)^{1 / n-1},(2 / \gamma)^{1 / n-1}\right)$ is the general escape criterion.

Proof. Using induction method on $\mathrm{n}$, For $n=1, G_{c}(z)=z+c$,

$$
|z|>\max (|c|, 0,0,0)
$$

For $n=2, G_{c}(z)=z^{2}+c$,

$$
|z|>\max (|c|, 2 / \alpha, 2 / \beta, 2 / \gamma)
$$

and for $n=3, G_{c}(z)=z^{3}+c$,

$$
|z|>\max \left(|c|,(2 / \alpha)^{1 / 2},(2 / \beta)^{1 / 2},(2 / \gamma)^{1 / 2}\right)
$$

Now, for $n+1$, let $G_{c}(z)=z^{n+1}+c$ and $|z| \geq|c|>(2 / \alpha)^{1 / n},|z| \geq|c|>$ $(2 / \beta)^{1 / n}$ and $|z| \geq|c|>(2 / \gamma)^{1 / n}$. Then

$$
\begin{aligned}
\left|G_{c}^{\prime}(z)\right| & =\left|(1-\gamma) z+\gamma G_{c}^{\prime \prime}(z)\right|, \text { where } G_{c}^{\prime \prime}(z)=z^{n+1}+c \\
& =\left|(1-\gamma) z+\gamma\left(z^{n+1}+c\right)\right| \\
& \geq \gamma|z|^{n+1}-(1-\gamma)|z|-\gamma|z| \quad \quad \quad(\text { since }|z| \geq|c|) \\
& =|z|\left(\gamma\left|z^{n}\right|-1\right),
\end{aligned}
$$




$$
\text { i.e. }\left|G_{c}^{\prime}(z)\right| \geq|z|\left(\gamma\left|z^{n}\right|-1\right) \text {. }
$$

Therefore

$$
\begin{aligned}
\left|G_{c}(z)\right| & =\left|(1-\beta) G_{c}^{\prime \prime}(z)+\beta G_{c}^{\prime}(z)\right| \\
& \geq\left|(1-\beta)\left(z^{n+1}+c\right)+\beta\right| z\left|\left(\gamma\left|z^{n}\right|-1\right)\right| \\
& \geq(1-\beta)|z|^{n+1}-(1-\beta)|z|+\beta \gamma|z|^{n+1}-\beta|z| \\
& \geq|z|\left[(\beta \gamma-\beta+1)|z|^{n}-1\right] \\
\text { i.e. } \quad\left|G_{c}(z)\right| & \geq|z|\left[(\beta \gamma-\beta+1)|z|^{n}-1\right] .
\end{aligned}
$$

Since $z_{n}=(1-\alpha) G_{c}\left(z_{n-1}\right)+\alpha G_{c}^{\prime}\left(z_{n-1}\right)$, we obtain

$$
\begin{aligned}
\left|z_{1}\right| & =\left|(1-\alpha) G_{c}(z)+\alpha G_{c}^{\prime}(z)\right| \\
& =|(1-\alpha)| z\left|\left[(\beta \gamma-\beta+1)|z|^{n}-1\right]+\alpha\right| z\left|\left(\gamma\left|z^{n}\right|-1\right)\right| \\
& \geq\left.\left|(|z|-\alpha|z|)\left[\beta \gamma|z|^{n}-\beta|z|^{n}+|z|^{n}-1\right]+\alpha \gamma\right| z\right|^{n+1}-\alpha|z| \mid \\
& \geq|z|\left[(\alpha \beta+\beta \gamma+\alpha \gamma-\alpha \beta \gamma-\beta-\alpha+1)|z|^{n}-1\right], \\
\left|z_{1}\right| & \geq|z|\left[(\alpha \beta+\beta \gamma+\alpha \gamma-\alpha \beta \gamma-\beta-\alpha+1)|z|^{n}-1\right] .
\end{aligned}
$$

Since $|z|>(2 / \alpha)^{1 / n},|z|>(2 / \beta)^{1 / n}$, and $|z|>(2 / \gamma)^{1 / n}$, so that $|z|>$ $(2 / \alpha \beta \gamma)^{1 / n}>(2 /(\alpha \beta+\beta \gamma+\alpha \gamma-\alpha \beta \gamma-\alpha-\beta-\gamma+1))^{1 / n}$.

Hence there exits a $\lambda>1$, such that $\left|z_{1}\right|>(1+\lambda)|z|$. Applying the inequality $\mathrm{n}$ times, we find $\left|z_{n}\right|>(1+\lambda)^{n}|z|$. Hence the result.

Corollary 3.8. (Escape Criterion). Suppose

$$
\left|z_{k}\right|>\max \left\{|c|,(2 / \alpha)^{1 / k-1},(2 / \beta)^{1 / k-1},(2 / \gamma)^{1 / k-1}\right\}
$$

for some $k \geq 0$, then $\left|z_{k}\right|>\lambda\left|z_{k-1}\right|$ and $\left|z_{n}\right| \rightarrow \infty$ as $|n| \rightarrow \infty$.

\section{Julia Sets}

In this section, various Julia sets have been generated for quadratic and cubic polynomials using new faster iteration process . 


\subsection{Julia sets for Quadratic Polynomials}

Following results have been observed from the graphical representation (see on page no. 15) of quadratic Julia sets:

1. It is observed that Figs 1-3, took the shape of Dragon Julia sets which has reflectional symmetry along x-axis.

2. The Dragon Julia sets get fattier with decrease in the parameters $\alpha, \beta$ and $\gamma$ and fixing the value of parameter c.

3. Further, Figs 4-6 shows connected Julia sets which have the reflectional symmetry about $\mathrm{x}$-axis and $\mathrm{y}$-axis.

4. Julia set in Fig. 7, shows the disconnectivity of orbits of Julia sets.

\subsection{Julia Sets for Cubic Polynomial}

In this section we study few cubic Julia sets. It is interesting to see that in Figs 10-12 (see on Page 16), cubic Julia sets took the shape of Lord Ganesha (name of a Hindu God). Further, it is observed that cubic Julia set becomes more connected (i.e. fattier) by decreasing the parameters $\alpha, \beta$ and $\gamma$ and fixing the value of parameter $c$.

\section{Mandelbrot Sets}

\subsection{Mandelbrot Sets for Quadratic Polynomials}

In this section we present few Mandelbrot sets using faster iteration process for quadratic, cubic and higher order polynomials. Following results have been analyzed from the graphical representation of Mandelbrot sets: Figs 16-24 (see on Page no. 17) shows the graphical representation of the quadratic Mandelbrot sets generated in computational software Mathematica. Here, we observed that the quadratic Mandelbrot sets have reflectional symmetry along x-axis (called line of symmetry). Further from Figs 16-18 (see on Page no. 17), it was analyzed that when any two parameters out of $\alpha, \beta$ and $\gamma$ are fixed then Mandelbrot sets get fattier with increase in the third parameter and becomes slimmer with decrease in the third parameter. From Figs 19-21 (see on Page no. 17), it was observed that when the value of parameter beta is increased, the lobes of the Mandelbrot sets get fattier. 


\subsection{Mandelbrot Sets for Cubic and Higher Order Polynomials}

In case of cubic polynomials, it was observed that there is a reflectional symmetry along x-axis and y-axis (see Figs 25-28 on Page no. 18). Further, we show that Figs 25-27 took the shape of fascinating Coupled Urn. As we decrease the parameters $\alpha, \beta$ and $\gamma$ they become more decorated Coupled Urns. In case of higher order polynomials, we observe that the polynomial $z^{n}+c$, $n>2$ generates the Mandelbrot sets that have $(n-1)$ lobes and also have reflectional (along $\mathrm{x}$-axis and $\mathrm{y}$-axis) and rotational symmetry (along center) (see Figs 30-32 on Page no. 18).

\section{Conclusions}

In this paper, a new faster iterative process have been studied for generalizations of Julia and Mandelbrot sets for complex quadratic, cubic and higher order polynomials. In Section 3, first we have presented new escape criterions for complex quadratic, cubic and higher order polynomials and then new Julia and Mandelbrot sets have been generated. From the graphical representations of Julia sets we have analyzed that few quadratic and cubic Julia sets took the shape of Dragon and Lord Ganesha (Hindu God name) respectively. Further, some Mandelbrot sets also took the shape of fascinating Urn.

\section{Acknowledgments}

This work is supported by the University Grant Commission of India (Grant No.F.173/2008(SA-1)). 


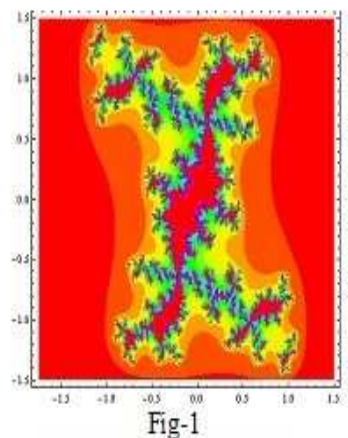

Dragon Julia set (A) for $\alpha=0.9, \beta=0.8, \gamma=0.7$, $c=0.5+0.5 \mathrm{I}$

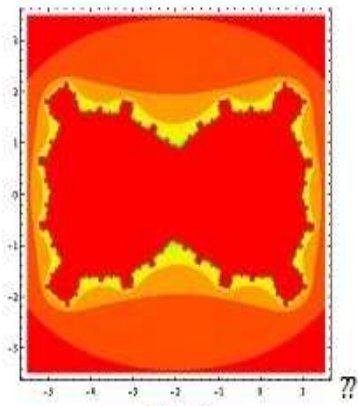

Fig-4

Quadratic Julia set for $\alpha=0.2, \beta=1, \gamma=1$, $c=0.05+0.05 \mathrm{I}$

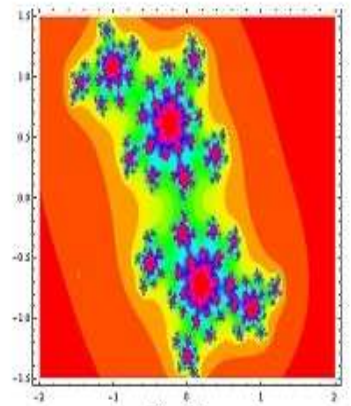

Fig-7

Quadratic Julia set for $\alpha=0.3, \beta=0.3, \gamma=0.3$, $c=0.1+0.9 \mathrm{I}$

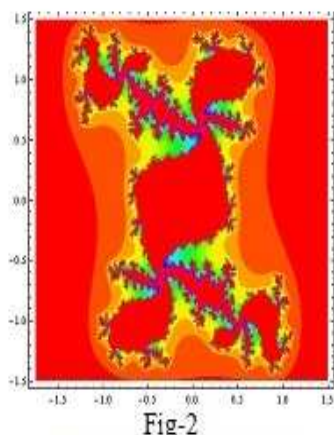

Dragon Julia set $(B)$ for $\alpha=0.8, \beta=0.9, \gamma=0.7$, $c=0.5+0.5 \mathrm{I}$

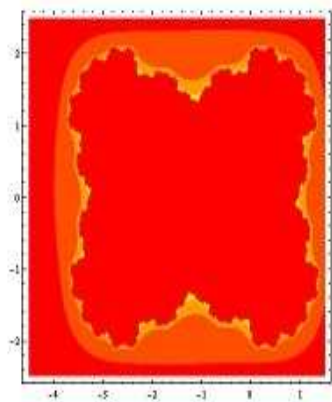

Fig-5

Quadratic Julia set for $\alpha=0.3, \beta=1, \gamma=0.8$, $c=0.1+0.1 \mathrm{I}$

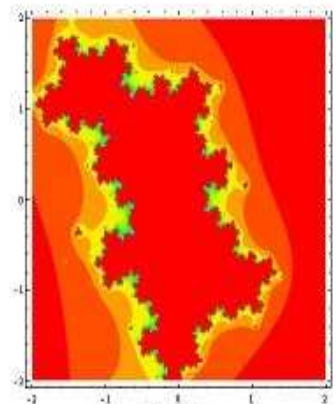

Fig-8

Quadratic Julia set for $\alpha=0.4, \beta=0.4, \gamma=0.4$, $c=0.1+0.9 \mathrm{I}$

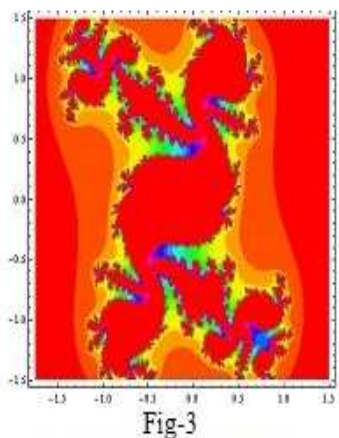

Dragon Julia set (C) for $\alpha=0.7, \beta=0.8, \gamma=0.6$, $c=0.5+0.5 \mathrm{I}$

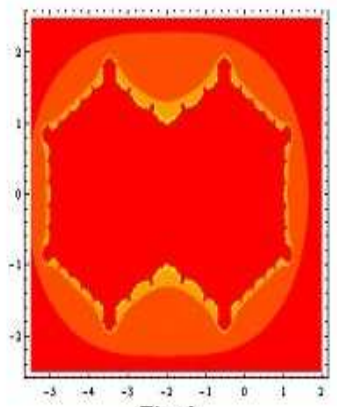

Fig-6

Quadratic Julia set for $\alpha=0.2, \beta=0.9, \gamma=0.2$, $c=0.01+0.01 \mathrm{I}$

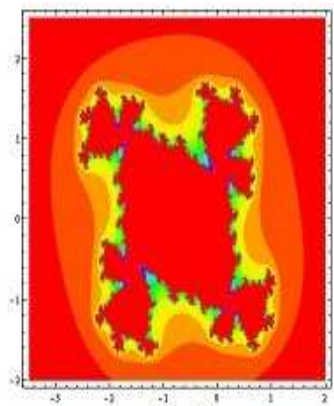

Fig-9

Quadratic Julia set for $\alpha=0.4, \beta=1.0, \gamma=1.0$, $c=0.1+0.9 \mathrm{I}$ 


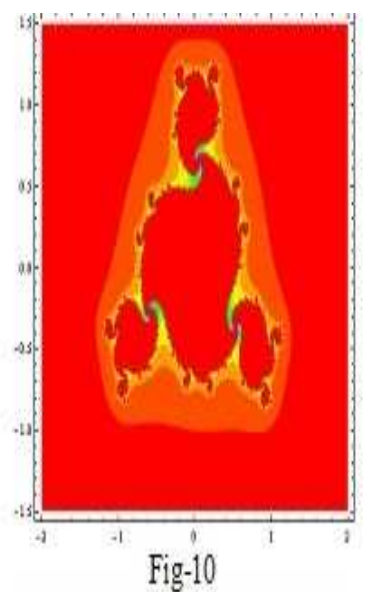

Lord Ganesha (A) Julia set for $\alpha=0.3, \beta=0.1, \gamma=0.1$, $c=0.1+0.9 I$

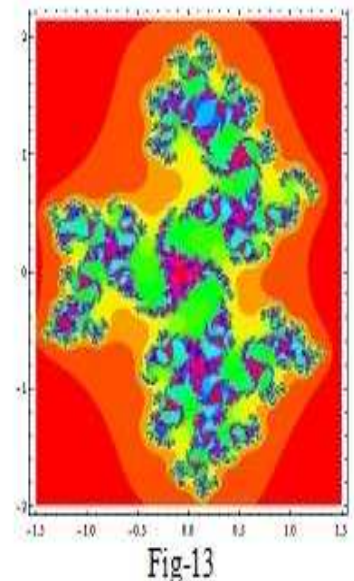

Cubic Julia set for $\alpha=0.1, \beta=0.7, \gamma=0.9$, $c=0.7+0.2 I$

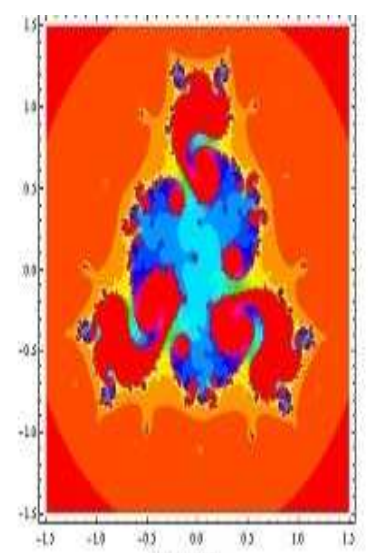

Fig-11 Lord Ganesha(B) Julia set for $\alpha=0.9, \beta=0.7, y=0.9$. $c=0.1+0.9 \mathrm{I}$

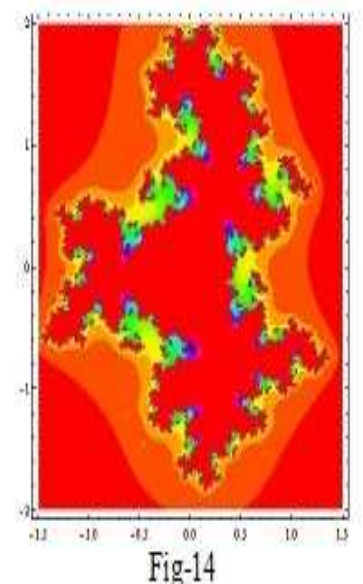

Cubic Julia set for $\alpha=0.1, \beta=0.7, \gamma=0.9$, $c=0.7+0.7 \mathrm{I}$

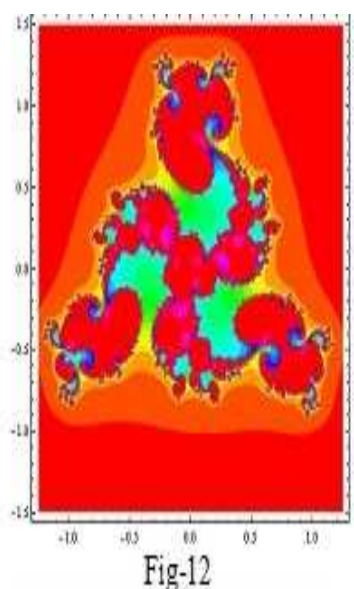

Lord Ganesha $(C)$ Julia set for $a=0.26, \beta=0.16, \gamma=0.26$, $c=0.11+0.95 I$

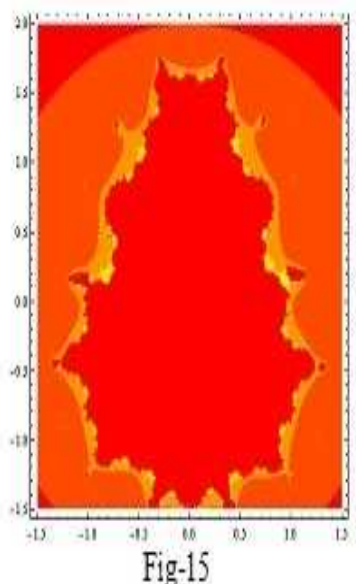

Cubic Julia set for $a=0.1, \beta=0.7, \gamma=0.9$, $c=0.1+0.9 \mathrm{I}$ 


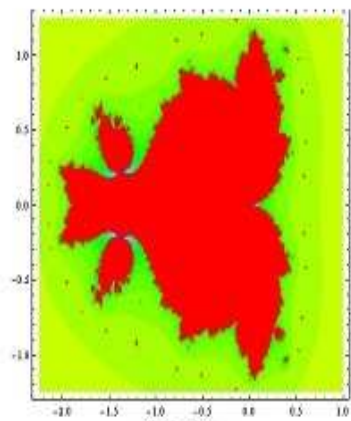

Fig.-16

Quadratic Mandelbrot set for $\alpha=0.3, \beta=0.1, \gamma=0.3$

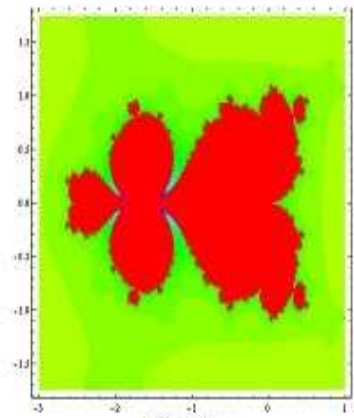

Fig. 19

Quadratic Mandelbrot set for $\alpha=0.1, \beta=0.8, \gamma=0.1$

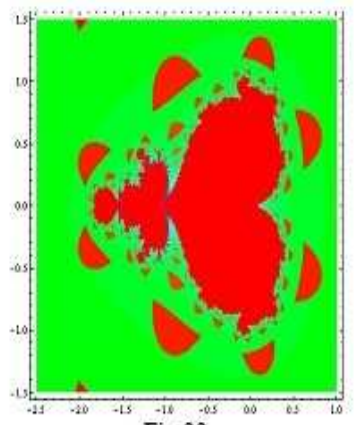

Fig. 22

Quadratic Mandelbrot set for $\alpha=1, \beta=0.1, \gamma=1$

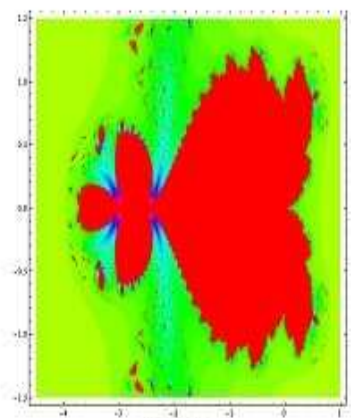

Fig. -17

Quadratic Mandelbrot set for

$\alpha=0.3, \beta=0.6, \gamma=0.3$

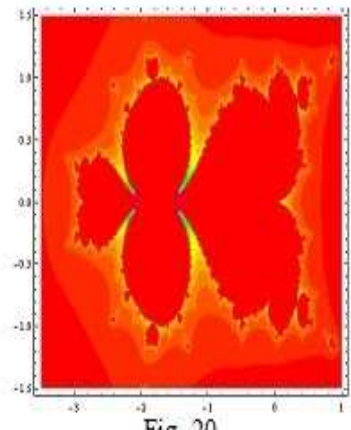

Fig. -20

Quadratic Mandelbrot set for $\alpha=0.1, \beta=0.9, \gamma=0.1$

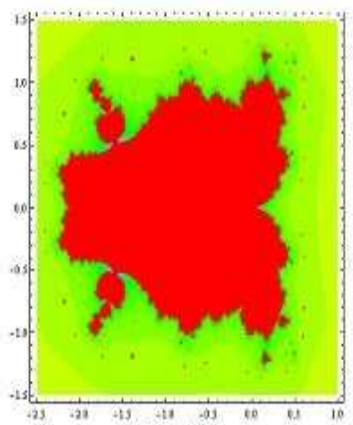

Fig. -23

Quadratic Mandelbrot set for $\alpha=0.2, \beta=03, \gamma=0.4$

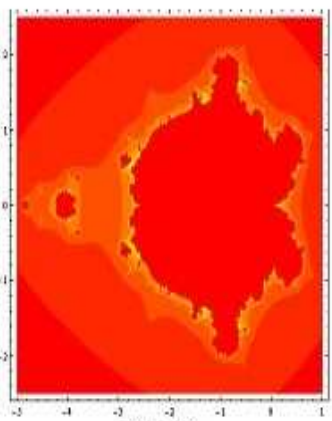

Fig. 18

Quadratic Mandelbrot set for

$\alpha=0.3, \beta=0.9, \gamma=0.3$

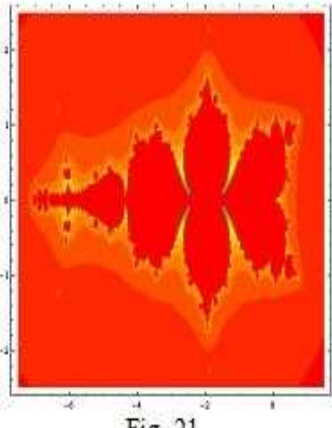

Fig.-21

Quadratic Mandelbrot set for $\alpha=0.1, \beta=1.0, \gamma=0.1$

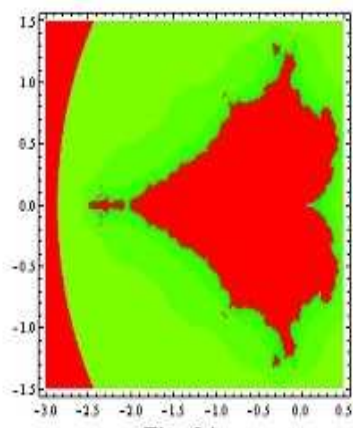

Fig. 24

Quadratic Mandelbrot set for $\alpha=0.7, \beta=0.8, \gamma=0.9$ 


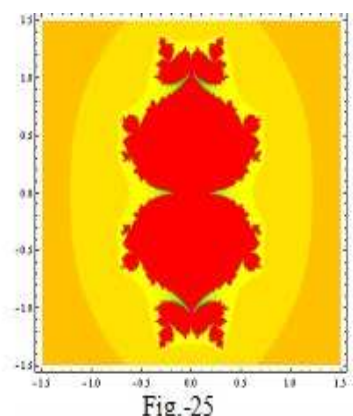

Coupled urn(A) Mandelbrot set for $\alpha=0.1, \beta=0.1, \gamma=0.1$

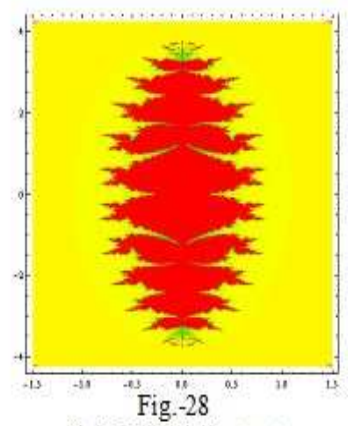

Cubic Mandelbrot set for $\alpha=0.1, \beta=0.9, \gamma=0.1$

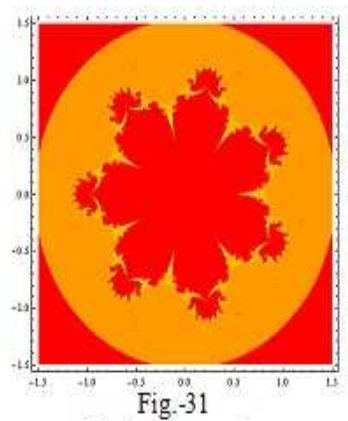

Mandelbrot set for $\alpha=0.1, \beta=0.3, \gamma=0.1$ for $n=8$

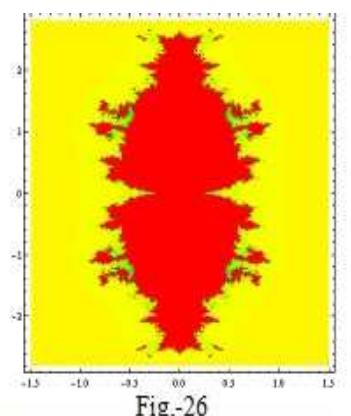

Coupled urn(B) Mandelbrot set For $\alpha=0.2, \beta=09, \gamma=0.2$

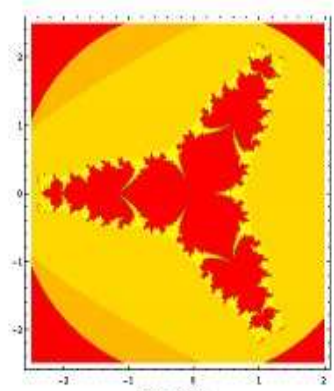

Fig. 29

Mandelbrot set for $\alpha=0.1, \beta=0.9, \gamma=0.1$ for $n=4$

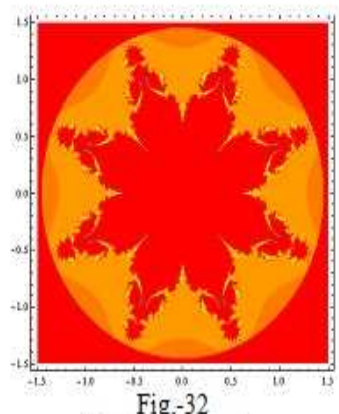

Mandelbrot set for $\alpha=0.1, \beta=0.9, \gamma=0.1$ for $n=9$

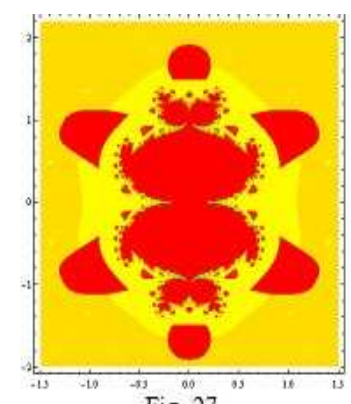

Fig.-27

Coupled urn(C) Mandelbrot set for $\alpha=0.9, \beta=0.1, \gamma=0.9$

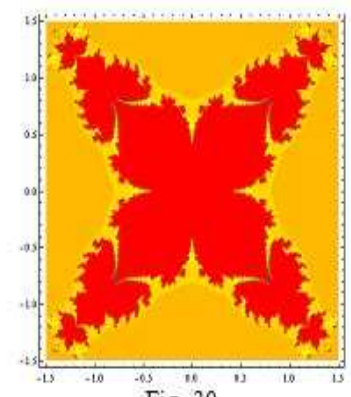

Fig. -30

Mandelbrot set for $\alpha=0.1, \beta=0.9, \gamma=0.1$ for $n=5$

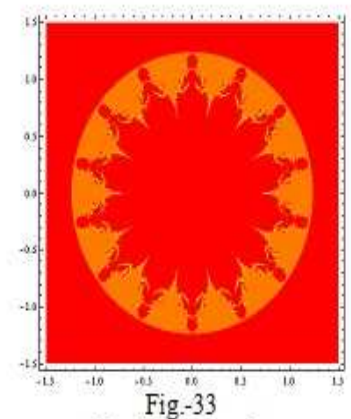

Mandelbrot set for $\alpha=0.1, \beta=0.9, \gamma=0.1$ for $n=15$ 


\section{References}

[1] A. Douady and J. H. Hubbard, tude dynamique des plynomes complexes, Publications Mathematiques dOrsay 84-02, ( 1984).

[2] A. Epstein and M. Yampolsky, Geography of the cubic connectedness locus: intertwining surgery, Ann. Sci. cole Norm. Sup., (32)(2)(1999), pp. 151-185.

[3] A. J. Crilly , R. A. Earnshaw and H. Jones, Fractals and Chaos, Springer-Verlag, New York (1991).

[4] A. Negi and M. Rani, A new approach to dynamic noise on superior Mandelbrot set, Chaos Solitons Fractals, (36)(4)(2008), pp. 1089-1096.

[5] A. Negi and M. Rani, Midgets of superior Mandelbrot set, Chaos Solitons Fractals, (36)(2)(2008), pp. 237-245.

[6] Ashish, M. Rani and R. Chugh, Dynamics of Antifractals in Noor orbit, J. Math. Anal. Appl., (57)(4)(2012), pp. 11-15.

[7] Ashish, M. Rani and R. Chugh, Study of cubic Julia sets in NO, International Journal of Computational Science, Engineering and Technology,,(1)(1)(2013), pp. 13-17.

[8] Ashish, M. Rani, and R. Chugh, Julia sets and Mandelbrot sets in Noor orbit, App. Math. Comput., (228)(2014), pp. 615-631.

[9] D. Rochon, A Generalized Mandelbrot set for bicomplex numbers, Fractals, (8)(4)(2000), pp. 355-368.

[10] H. O. Peitgen, H. Jurgens and D. Saupe, Chaos and Fractals,, Springer-Verlag, New York, Inc., (1992).

[11] M. Frame and Robertson J.,A generalized mandelbrot set and the role of critical points, Computer and Graphics,(16)(1)(1992), pp. 35-40.

[12] M. Abbas and T. Nazir, A new iteration process applied to constained minimization and feasibility problems, Matemathykm Bechnk (66)(2) (2014), pp. 223-234.

[13] M. Rani, Superior tricorns and multicorns, in: WSEAS Proc. 9th Int. conf. on Applications of Computer Engineering, (ACE 10): Recent Advances and Applications of Computer Engineering,(2010), pp. 58-61.

[14] M. Rani and R. Agarwal, Effect of noise on Julia sets generated by logistic map, in: Proc. 2nd IEEE International Conference on Computer and Automation Engineering,(2)(2010), pp. 55-59.

[15] M. Rani and R. Agarwal, Effect of stochastic noise on superior Julia sets, J. Math. Imaging Vis.,(36)(2010), pp. 63-68.

[16] M. Rani and M. Kumar, Circular saw Mandelbrot sets, in: WSEAS Proc. 14th Int. conf. on Applied Mathematics (Math 09): Recent Advances in Applied Mathematics,(2009), pp. 131-136.

[17] M. Rani and V. Kumar, Superior Julia set, J. Korean Soc. Math. Edu. Res. Ser. $D,(8)(4)(2004)$, pp. 261-277.

[18] M. Rani and V. Kumar, Superior Mandelbrot set, J. Korean Soc. Math. Edu. Res. Ser. $D,(8)(4)(2004)$, pp. 279-291.

[19] R. Agarwal and V. Agarwal, Dynamic noise perturbed generalized superior Mandelbrot sets, Nonlinear Dyn., (67)(3) (2011), pp. 1883-1891. 
[20] R. Chugh and Ashish, Fractals generated by various iterative procedures - A Survey, Mathematical Journal of Interdisciplinary Sciences, (2)(2)(2014), pp. 195-208.

[21] R. L. Devaney, A First Course in Chaotic Dynamical Systems: Theory and Experiment, Addison-Wesley, (1992).

[22] S. S. Liaw, The parameter spaces of the cubic polynomials, Fractals,, (6)(2)(1998), pp. 181-189.

[23] S. L. Singh, S. N. Mishra and W. Sinkala, A new iterative approach to fractal models, Communications in Nonlinear Science and Numerical Simulations,(17)(2)(2011), pp. 521529.

[24] T. Lei, Similarity between the Mandelbrot setsand Julia sets, Commun. Math. Phys.,, (134)(3)(1990), pp. 587-617.

[25] X. Wang and C. Luo, Generalized Julia sets from a non-analytic complex mapping, Appl. Math. Comput.,(181)(2006), pp. 113-122.

[26] Y. H. Geum and K. G. Hare, Groebner basis, resultants and the generalized Mandelbrot set, Chaos, Solitons, Fractals ,(42)(2)(2009), pp. 1016-1023.

[27] Y. Sun and X. Wang, Noise perturbed quaterionic Mandelbrot sets, Int. J. Comp. Sci.,(86)(12)(2009), pp. 2008-2028. 
